\title{
Reviving the interference: Framework and proof-of-principle for the anomalous gluon self-interaction in the SMEFT
}

\author{
Céline Degrande ${ }^{*}$ and Matteo Maltoni® ${ }^{\dagger}$ \\ Centre for Cosmology, Particle Physics and Phenomenology (CP3), Université catholique de Louvain, \\ 1348 Louvain-la-Neuve, Belgium
}

(Received 6 January 2021; accepted 22 April 2021; published 12 May 2021)

\begin{abstract}
Interferences are not positive-definite and therefore they can change sign over the phase space. If the contributions of the regions where the interference is positive and negative nearly cancel each other, interference effects are hard to measure. In this paper, we propose a method to quantify the ability of an observable to separate an interference positive and negative contributions and therefore to revive the interference effects in measurements. We apply this method to the anomalous gluon operator in the SMEFT for which the interference suppression is well-known. We show that we can get, for the first time, constraints on its coefficient using the interference only similar to those obtained by including the square of the new physics amplitude.
\end{abstract}

DOI: 10.1103/PhysRevD.103.095009

\section{INTRODUCTION}

The Standard Model effective field theory (SMEFT) explores the deviations in SM couplings due to interactions among Standard Model (SM) particles and new states, too heavy to be produced at the LHC or any other considered experiment. Nonetheless, those new states affect the interactions between the SM particles and accurate measurements of their strengths should, thus, reveal or constrain the presence of new physics. In this framework, heavy new degrees of freedom are integrated out and the new physics is parametrized by higher-dimensional operators $[1,2]$,

$$
\mathcal{L}_{\mathrm{SMEFT}}=\mathcal{L}_{\mathrm{SM}}+\sum_{i} \frac{C_{i}}{\Lambda^{2}} O_{i}+\mathcal{O}\left(\Lambda^{-4}\right)
$$

where $\Lambda$ is the new physics scale. As a results, observables such as differential cross sections display the same expansion,

$$
\frac{d \sigma}{d X}=\frac{d \sigma^{\mathrm{SM}}}{d X}+\sum_{i} \frac{C_{i}}{\Lambda^{2}} \frac{d \sigma}{d X}+\mathcal{O}\left(\Lambda^{-4}\right)
$$

where $\mathrm{X}$ is a generic name for a measurable variable. While constraints should ideally come from the second term,

\footnotetext{
*celine.degrande@uclouvain.be

†matteo.maltoni@uclouvain.be
}

Published by the American Physical Society under the terms of the Creative Commons Attribution 4.0 International license. Further distribution of this work must maintain attribution to the author(s) and the published article's title, journal citation, and DOI. Funded by SCOAP ${ }^{3}$. i.e., the term linear in the coefficients, they often come in practice from the term quadratic in $C_{i}$ or from terms of even higher power of $C_{i}$. This phenomenon mainly originates from the fact that the linear term is an interference between the SM amplitudes and the amplitudes linear in $C_{i}$, and this interference has been shown to be suppressed [3] for $2 \rightarrow 2$ processes. As it will be illustrated below, this suppression occurs also in higher multiplicity processes. An interference suppression can have two origins: either the interference matrix element is small all over the phase space, or it changes sign over the phase space. This paper aims, in the second case, to revive the interference using differential measurements and to assess the efficiency of the reviving procedure. Although we will focus on a single operator in the rest of the paper, the method is generic and can be applied for any interference suppressed by a sign flip in the phase space, including interference unrelated to the SMEFT. Another obvious application in the SMEFT is the $C P$-violating operators [4]. Their interference do not contribute to the total cross section of $\mathrm{C}$-even processes by symmetry, but they can probed using $C P$-violating observables.

\section{FRAMEWORK}

In this work we concentrate on the dimension- 6 operator

$$
O_{G}=g_{s} f_{a b c} G_{\nu}^{a, \mu} G_{\rho}^{b, \nu} G_{\mu}^{c, \rho},
$$

with $G_{\mu \nu}$ the gluon field strength. While this operator is expected to contribute to multijets and top-pair production, its interference vanishes for dijet and is strongly suppressed for the other processes. As a matter of fact, previous 
studies [5-7] suggest that a good sensitivity to its interference is unachievable. However, constraints on this operator are essential as they affect the sensitivity over other operators involved, for example, in top quark production [8]. High-multiplicity jet measurements strongly constrain this operator but mainly from the $\mathcal{O}\left(\Lambda^{-4}\right)$ or even higher order terms $[6,7]$. The stricter bound on this operator comes from the $\mathcal{O}\left(\Lambda^{-4}\right)$ in dijet measurements [9] and reads

$$
\frac{C_{G}}{\Lambda^{2}}<(0.031 \mathrm{TeV})^{-2}
$$

at 95\% confidence level (CL). We use the SMEFT@NLO [10] Universal FeynRules Output (UFO) [11], written from a FeynRules model [12] containing the dimension-six operators, to generate the LO partonic events needed for our study. All the operators coefficients are set to zero but the $O_{G}$ one, which is taken equal to 1 with $\Lambda=5 \mathrm{TeV}$. Madgraph@NLO [13] is then used to generate events for the SM, the square of the $1 / \Lambda^{2}$ amplitudes and their interference. Throughout this paper, we truncate the amplitude at $\mathcal{O}\left(1 / \Lambda^{2}\right)$ and therefore $\mathcal{O}\left(1 / \Lambda^{4}\right)$ terms always come from the square of the $1 / \Lambda^{2}$ amplitudes. Namely, multiple insertions of the dimension-six operators are not allowed. We use the NNPDF2.3 parton distribution function (PDF) set [14] and the results are given for LHC at $13 \mathrm{TeV}$ at the partonic level. We leave the study of the effect of NLO corrections, parton shower and detector effects for future studies. The cancellation over the phase space is efficient if the integrals of the interference in the phase space part where its matrix element is positive and negative are almost equal in absolute value. Those two integrals are obtained from the sum of the weights of events generated according to the interference, keeping respectively only positive or negative weighted events. In Table I, we use the percentage of positive unweighted events to quantify the efficiency of this cancelation for top and jet processes. Since the strongest cancellation occurs for three-jets and this process has the large cross section necessary for accurate differential measurement, in the remaining of this paper, we will restrict ourself to this process and leave the other for future analyses. The integral of the absolute valued interference differential cross section,

$$
\sigma^{|\mathrm{int}|} \equiv \int d \Phi\left|\frac{d \sigma_{\text {int }}}{d \Phi}\right|
$$

is computed from the sum of the absolute values of the weights and is an upper bound of the total measurable effect of the interference over the whole phase space $\Phi$. This quantity is given in Table II together with the SM, the interference and the $\mathcal{O}\left(1 / \Lambda^{4}\right)$ total cross-sections. The comparison of those four quantities shows the strong suppression of the interference total cross section, and how it is lifted by $\sigma^{\text {int }}$. Unfortunately, $\sigma^{\text {int } \mid}$ is not a measurable quantity as it requires to measure not only the momenta of the jets, but also their flavors and helicities, as well as those of the incoming partons. Therefore, we define the measurable absolute value cross section,

$$
\sigma^{\mid \text {meas } \mid} \equiv \int d \Phi_{\text {meas }}\left|\sum_{\{u m\}} \frac{d \sigma}{d \Phi}\right|
$$

where $\{u m\}$ is the set of unmeasurable quantities of the events. For other processes, the sum can be replaced, at least partially, by integrals over continuous unmeasurable quantities, such as the longitudinal momenta of a neutrino. This is the difference between the positive and negative contributions of the interference to the total cross section using all the information experimentally available (and assuming perfect measurements of the jets momenta). As a result, this is an upper bound for any asymmetry build on one or a few kinematic variables aiming at restoring the interference, and therefore can be used to assess the efficiency of such asymmetry. $\sigma^{\mid \text {meas } \mid}$ is estimated by

$$
\sigma^{\mid \text {meas } \mid}=\lim _{N \rightarrow \infty} \sum_{i=1}^{N} w_{i} * \operatorname{sign}\left(\sum_{u m} \operatorname{ME}\left(\vec{p}_{i}, u m\right)\right)
$$

where $\mathrm{ME}$ is the part of the squared amplitude due to the interference and $w_{i}$ and $\vec{p}_{i}$ label the weight and the momenta of the jets of the event $i$. Therefore, this can be seen as a matrix element method [15-20] at the partonic

\begin{tabular}{|c|c|c|c|c|c|c|}
\hline \multirow[b]{2}{*}{ Proc. } & \multicolumn{2}{|c|}{$p_{T}>50 \mathrm{GeV}$} & \multicolumn{2}{|c|}{$p_{T}>200 \mathrm{GeV}$} & \multicolumn{2}{|c|}{$p_{T}>1000 \mathrm{GeV}$} \\
\hline & $\sigma[\mathrm{pb}]$ & $w>0$ & $\sigma[\mathrm{pb}]$ & $\mathrm{w}>0$ & $\sigma[\mathrm{pb}]$ & $w>0$ \\
\hline$t \bar{t}$ & 1.384 & $85 \%$ & 1.384 & $85 \%$ & 1.384 & $85 \%$ \\
\hline$t \bar{t} j$ & $5.20 \times 10^{-1}$ & $62 \%$ & $1.13 \times 10^{-1}$ & $60 \%$ & $1.37 \times 10^{-3}$ & $62 \%$ \\
\hline$j j j$ & $2.98 \times 10^{1}$ & $52 \%$ & $5.90 \times 10^{-1}$ & $52 \%$ & $4.91 \times 10^{-4}$ & $61 \%$ \\
\hline$j j j j$ & $-2.89 \times 10^{1}$ & $45 \%$ & $-2.50 \times 10^{-1}$ & $44 \%$ & $-4.12 \times 10^{-6}$ & $39 \%$ \\
\hline
\end{tabular}

TABLE I. $\mathcal{O}\left(\Lambda^{-2}\right)$ cross sections and percentages of positive-weighted events for processes with a non-null interference between the $\mathrm{SM}$ and the $O_{G}$ operator and a large cross section. These results are calculated for jets separated by $\Delta R>0.4$ and with different minimum values for their transverse momentum $p_{T}$. 
TABLE II. Cross sections for three-jet production, for different values of the $p_{T}$-cut, $\Delta R>0.4, \Lambda=5 \mathrm{TeV}$ and renormalization scales fixed respectively at $150,250,500,1000$ and $2000 \mathrm{GeV}$, with up to one $O_{G}$ insertion. The percentages of the total amount of positive-weighted events, the percentages of the positive and negative measurable matrix elements (mme) and $\sigma^{\text {int } \mid}$ are shown for the interference.

\begin{tabular}{lcccccc}
\hline \hline & & \multicolumn{3}{c}{$\mathcal{O}\left(1 / \Lambda^{2}\right)$} & $\mathcal{O}\left(1 / \Lambda^{4}\right)$ \\
\cline { 3 - 6 }$p_{T, \text { min }}[\mathrm{GeV}]$ & $\sigma[\mathrm{pb}]$ & $\sigma[\mathrm{pb}]$ & $\operatorname{wgt}>0$ & $\sigma^{\mid \text {meas } \mid}[\mathrm{pb}]$ & $\sigma^{\mid \text {int } \mid}[\mathrm{pb}]$ & $\sigma[\mathrm{pb}]$ \\
\hline 50 & $9.70 \times 10^{5}$ & 4.08 & $50.4 \%$ & $7.83 \times 10^{2}$ & $1.05 \times 10^{3}$ & $3.93 \times 10^{1}$ \\
200 & $8.96 \times 10^{2}$ & $2.92 \times 10^{-1}$ & $51.4 \%$ & $3.5 \times 10^{1}$ & $5.02 \times 10^{1}$ & 2.73 \\
500 & 3.10 & $1.69 \times 10^{-2}$ & $54.0 \%$ & $6.04 \times 10^{-1}$ & $8.96 \times 10^{-1}$ & $1.48 \times 10^{-1}$ \\
1000 & $9.08 \times 10^{-3}$ & $4.56 \times 10^{-4}$ & $60.1 \%$ & $1.46 \times 10^{-3}$ & $2.29 \times 10^{-3}$ & $3.05 \times 10^{-3}$ \\
\hline \hline
\end{tabular}

level to revive the interference. The values of $\sigma^{\mid \text {meas } \mid}$ for the three-jet final state and different cuts are given in Table II. The cancellation among positive and negative weighted events decreases with the $p_{T}$ cut while the ratio $\sigma^{\mid \text {meas } \mid} / \sigma^{\mid \text {int } \mid}$ remains roughly constant.

\section{DIFFERENTIAL DISTRIBUTIONS}

We tested the ability to separate positive and negative weight for various differential and double differential cross sections. Tested distributions include the transverse momenta $p_{T}$ and the pseudorapidities $\eta$ of the jets, their angular distances $\Delta R$, their invariant masses, the normalised triple product among the three-momenta of the jets, and some event-shape variables, including the transverse thrust, the jet broadening [21] and the transverse sphericity [22]. Several variables such as the $p_{T}$ of the first jet, $p_{T}\left[j_{1}\right]$, the transverse trust and the angular distance between the two lowest $p_{T}$ jets, $\Delta R\left[j_{2} j_{3}\right]$ achieve an efficiency of about $40 \%$ compared to $\sigma^{\mid \text {meas } \mid}$. For comparison, the efficiency of the total cross section is about $2 \%$. The best efficiency, however, is obtained for the transverse sphericity and is about $80 \%$. Moreover, this efficiency barely varies with the global lower cut on each of the three jets $p_{T}$. The transverse sphericity $S p h_{T}$ is defined by using the eigenvalues $\lambda_{1} \geq \lambda_{2}$ of the transverse momentum tensor:

$M_{x y}=\sum_{i=1}^{N_{\mathrm{jets}}}\left(\begin{array}{cc}p_{x, i}^{2} & p_{x, i} p_{y, i} \\ p_{y, i} p_{x, i} & p_{y, i}^{2}\end{array}\right), \quad S p h_{T}=\frac{2 \lambda_{2}}{\lambda_{2}+\lambda_{1}}$.

Therefore, sign flip occurs between the events that are more two-jets like $\left(S p h_{T} \sim 0\right)$ and those that are three well separated and balanced jets $\left(S p h_{T} \sim 1\right)$. This explains why the phase space cancellation is lower with the high $p_{T}$ cut, as strong hierarchy between the jets becomes then unlikely. The separations of the negative and positive contributions for some of those variables are illustrated in Fig. 1, where the full distributions as well as those of the positive and negative weighted events are drawn separately. Contrarily to inefficient variables, the distribution of the positive and negative weighted events are different, resulting in a nonzero and changing sign distribution for the full interference.

NLO predictions for the interferences of operators known for their cancelation over the phase space seem
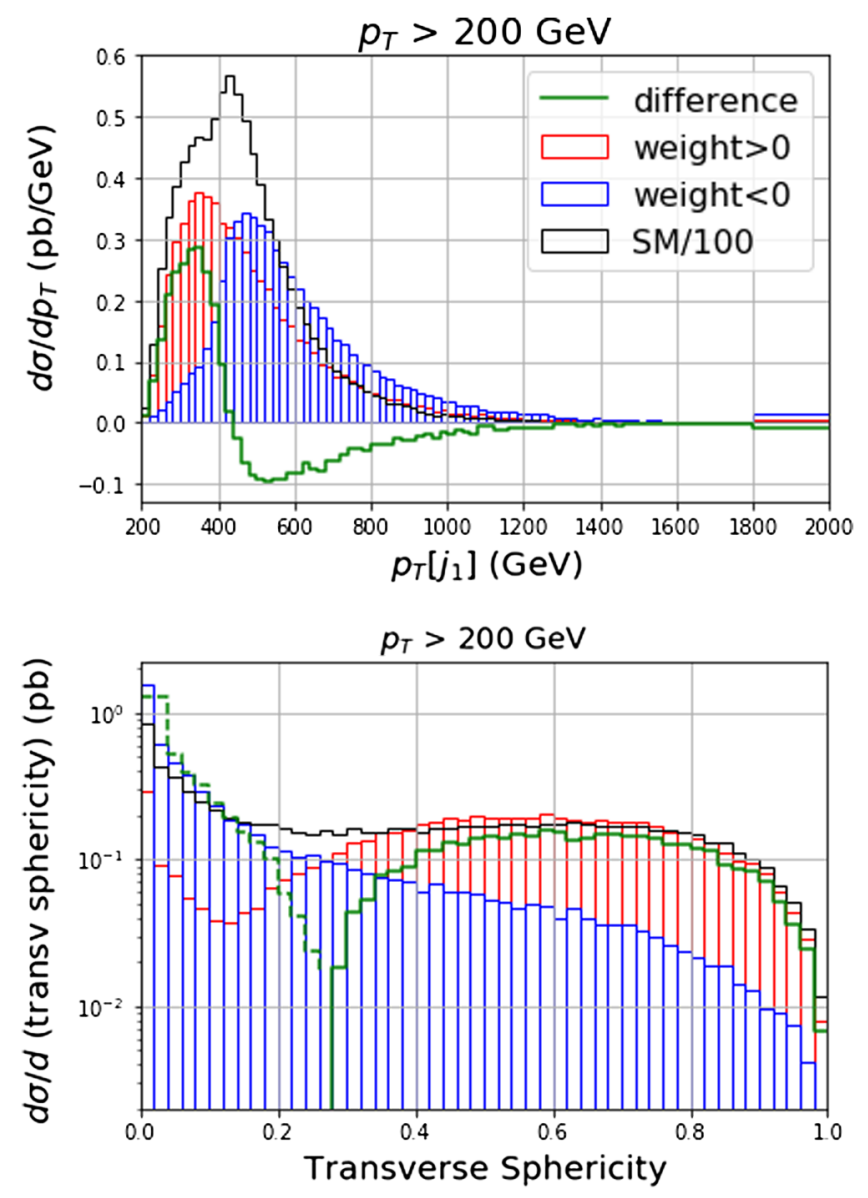

FIG. 1. Differential distributions for $p p \rightarrow 3 j$ at the LHC with $p_{T}>200 \mathrm{GeV}$ for the jets. The red (blue) line represents the differential cross-section contribution by the positive (negative) weighted events. Their difference, the green line, is the differential cross-section distribution for the interference; the dashed portion is the opposite of the negative differential distribution. The black line reproduces the SM cross-section distribution, divided by 100 . The last bins contain the overflow. 
Lost events for interference (\%)

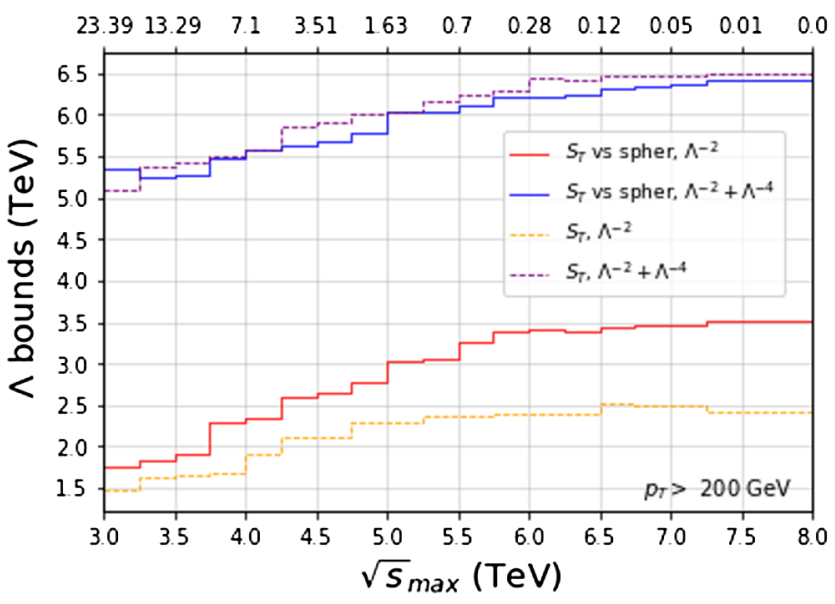

Lost events for interference (\%)

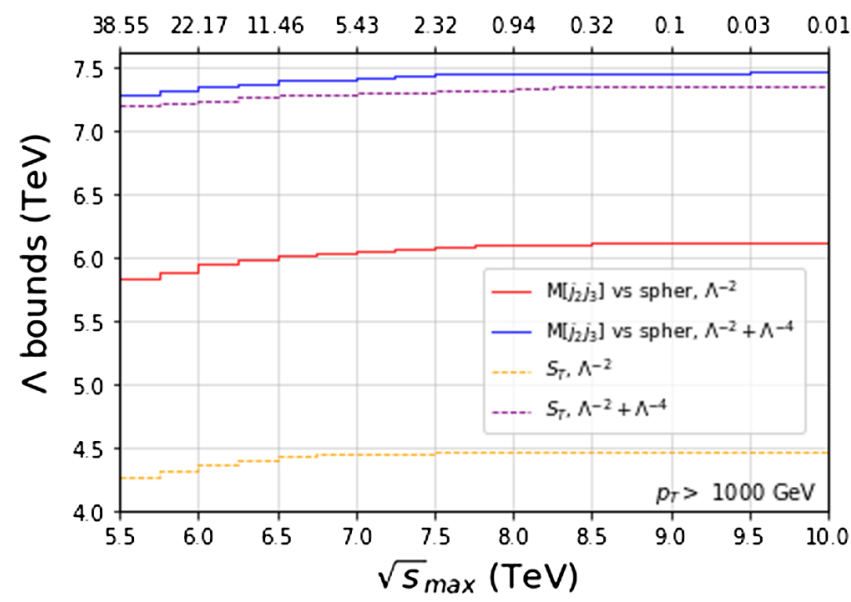

FIG. 2. Upper bounds on $\Lambda$ (for $C_{G}=1$ ) as functions of the upper cut over the center-of-mass energy $\sqrt{s}$, inferred from the best distribution for each $p_{T}$-cut. The red line shows the bounds from the $\mathcal{O}\left(\Lambda^{-2}\right)$ term only, which are symmetrical with respect to 0 , while the blue line take into account the $\mathcal{O}\left(\Lambda^{-4}\right)$ one, too. The orange and purple lines reproduce the bounds, obtained through the $S_{T}$ variable, considered in [6], at $\mathcal{O}\left(\Lambda^{-2}\right)$ and $\mathcal{O}\left(\Lambda^{-4}\right)$. The axis on top of the plots quantifies the percentage of events, in the interference sample, that get lost form the cut on $\sqrt{s}$. to lead in general to very large and/or negative $\mathrm{K}$-factors [4], as it is the case for analogous weak version of $O_{G}$, i.e., $O_{W}$. They can be understood by the fact that regions contributing positively and negatively to the interference have much more reasonable but different K-factors which can, therefore, significantly affect the level of cancelation. As a result, only observables able to separate the two regions would have stable predictions for the interference and would be able extract meaningful information about such interferences. Due to the heavy computation needed, we leave, however, the computation of the NLO corrections for our observables for future work.

Using the transverse sphericity to split the positive and negative contributions, we now estimate the limits that could be obtained on $\frac{C_{G}}{\Lambda^{2}}$, either for the interference only or including the $\mathcal{O}\left(1 / \Lambda^{4}\right)$ contribution, too. The bounds are obtained, for each double distribution, from the following $\chi$-squared

$$
\chi^{2}=\sum_{i}\left(\frac{x_{i}^{\mathrm{exp}}-x_{i}^{\mathrm{th}}}{\sigma_{i}}\right)^{2}=\sum_{i}\left(\frac{C_{G}}{\Lambda^{2}} x_{i}^{1 / \Lambda^{2}}\right)^{2}
$$

where $x_{i}^{\exp }$ and $x_{i}^{\text {th }}=x_{i}^{\mathrm{SM}}+\frac{C_{G}}{\Lambda^{2}} x_{i}^{1 / \Lambda^{2}}$ are respectively the measured and predicted content of each bin. Since the experimental results for the distributions we are interested in have not been published yet, we assume that the experimental data will follow the SM distributions for the considered quantities [resulting in the last step of Eq. (9)] and that the uncertainty, $\sigma_{i}$, for the $\mathrm{i}^{\text {th }}$ bin is $10 \%$ of its SM content. This estimate of the uncertainty seems consistent with available experimental results [23]. We choose our binning such that each bin would contain enough events, assuming the SM only to ensure that the statistical errors are below $10 \%$, for a luminosity of $100 \mathrm{fb}^{-1}$. The best results are displayed in Table III.

Finally, to assess the validity of the SMEFT with our approach, we display in Fig. 2 how the limits on $\Lambda$ varies if a cut on the center-of-mass energy is applied, assuming $C_{G}=1$. In principle, the EFT is valid if $\sqrt{s}<\Lambda$, which is only satisfied for $C_{G}$ slightly bigger that 1 with the low $p_{T}$

TABLE III. Best bounds on the $C_{G}$ coefficient for different cuts on the $p_{T}$, for $\Lambda=1 \mathrm{TeV}$ and $68 \%$ CL. The number of bins is reported, for each distribution; the cut on the sphericity is the value, between 0 and 1 , in which we separated the two bins used for this variable. In the bounds columns, the first numbers are obtained through the $\mathcal{O}\left(\Lambda^{-2}\right)$ contribution only, the ones into brackets take into account the $\mathcal{O}\left(\Lambda^{-4}\right)$ data, too.

\begin{tabular}{lccccc}
\hline \hline$p_{T, \text { min }}[\mathrm{GeV}]$ & Distribution & $S p h_{T}$ cut & Bins & Upper bound on $C_{G}$ & Lower bound on $C_{G}$ \\
\hline 50 & $p_{T}\left[j_{3}\right]$ vs $S p h_{T}$ & 0.23 & 34 & $2.5 \times 10^{-1}\left(1.1 \times 10^{-1}\right)$ & $-2.5 \times 10^{-1}\left(-1.2 \times 10^{-1}\right)$ \\
200 & $S_{T}$ vs $S p h_{T}$ & 0.27 & 34 & $7.5 \times 10^{-2}\left(2.3 \times 10^{-2}\right)$ & $-7.5 \times 10^{-2}\left(-2.4 \times 10^{-2}\right)$ \\
500 & $M\left[j_{2} j_{3}\right]$ vs $S p h_{T}$ & 0.31 & 21 & $5.5 \times 10^{-2}\left(5.3 \times 10^{-2}\right)$ & $-5.5 \times 10^{-2}\left(-3.5 \times 10^{-2}\right)$ \\
1000 & $M\left[j_{2} j_{3}\right]$ vs $S p h_{T}$ & 0.35 & 7 & $2.6 \times 10^{-2}\left(1.9 \times 10^{-2}\right)$ & $-2.6 \times 10^{-2}\left(-1.8 \times 10^{-2}\right)$ \\
\hline \hline
\end{tabular}


cuts. The situation improve for the stronger constraints derived with higher cuts. In both cases, the constraints barely change when the events with $\sqrt{s} \gtrsim 6 \mathrm{TeV}$ are included. The bounds, obtained through the interference only, grow faster than the ones which involve the $\mathcal{O}\left(\Lambda^{-4}\right)$ contribution too, as it is expected because of their different dependency on $\Lambda$. The bounds obtained by using the $S_{T}$ variable, defined in [6], are also shown for comparison. As expected, our distribution shows a nice improvement for the bounds at $\mathcal{O}\left(\Lambda^{-2}\right)$.

\section{CONCLUSIONS}

We used the sign of the measurable matrix element as a tool to revive the interference and to quantify the efficiency of differential distributions to separate negatively and positively contributing regions of the phase space. We used it to find efficient distributions to look for the interference effect of anomalous gluon interactions, as predicted by the SMEFT, and to put on the corresponding operators, for the first time, constraints which are dominated by the leading $\left[\mathcal{O}\left(\Lambda^{-2}\right)\right]$ interference and not by the $\mathcal{O}\left(\Lambda^{-4}\right)$ term, coming from the new physics amplitude squared. Therefore, we have finally found an answer to the long-standing quest for a sensitivity to the interference between the anomalous gluon operator and the SM. Due to its sensitivity to the interference, our observable is also sensitive to the sign of its coefficient. Finally, the proposed measurement can be easily reinterpreted in other BSM scenarios if SMEFT assumptions turn out not to be valid, as they are purely kinematic distributions. While the method has been tested on this particular case, it is fully generic and can be applied for any interference suppression due to sign flips over the phase space.

\section{ACKNOWLEDGMENTS}

We are grateful to Fabio Maltoni and Vincent Lemaitre for interesting discussions during the completion of this work. This work was funded by the F. R. S.-FNRS through the MISU convention F.6001.19.
[1] B. Grzadkowski, M. Iskrzynski, M. Misiak, and J. Rosiek, Dimension-six terms in the standard model Lagrangian, J. High Energy Phys. 10 (2010) 085.

[2] W. Buchmuller and D. Wyler, Effective Lagrangian analysis of new interactions and flavor conservation, Nucl. Phys. B268, 621 (1986).

[3] A. Azatov, R. Contino, C. S. Machado, and F. Riva, Helicity selection rules and noninterference for BSM amplitudes, Phys. Rev. D 95, 065014 (2017).

[4] C. Degrande and J. Toucheque (to be published).

[5] L. J. Dixon and Y. Shadmi, Testing gluon selfinteractions in three jet events at hadron colliders, Nucl. Phys. B423, 3 (1994); Erratum, Nucl. Phys. B452, 724 (1995).

[6] F. Krauss, S. Kuttimalai, and T. Plehn, LHC multijet events as a probe for anomalous dimension-six gluon interactions, Phys. Rev. D 95, 035024 (2017).

[7] V. Hirschi, F. Maltoni, I. Tsinikos, and E. Vryonidou, Constraining anomalous gluon self-interactions at the LHC: a reappraisal, J. High Energy Phys. 07 (2018) 093.

[8] A. Buckley, C. Englert, J. Ferrando, D. J. Miller, L. Moore, M. Russell, and C.D. White, Constraining top quark effective theory in the LHC Run II era, J. High Energy Phys. 04 (2016) 015.

[9] R. Goldouzian and M. D. Hildreth, LHC dijet angular distributions as a probe for the dimension-six triple gluon vertex, Phys. Lett. B 811, 135889 (2020).

[10] C. Degrande, G. Durieux, F. Maltoni, K. Mimasu, E. Vryonidou, and C. Zhang, Automated one-loop computations in the SMEFT, arXiv:2008.11743.

[11] C. Degrande, C. Duhr, B. Fuks, D. Grellscheid, O. Mattelaer, and T. Reiter, UFO - The universal feynrules output, Comput. Phys. Commun. 183, 1201 (2012).
[12] A. Alloul, N. D. Christensen, C. Degrande, C. Duhr, and B. Fuks, FeynRules 2.0-A complete toolbox for tree-level phenomenology, Comput. Phys. Commun. 185, 2250 (2014).

[13] J. Alwall, R. Frederix, S. Frixione, V. Hirschi, F. Maltoni, O. Mattelaer, H.-S. Shao, T. Stelzer, P. Torrielli, and M. Zaro, The automated computation of tree-level and next-toleading order differential cross sections, and their matching to parton shower simulations, J. High Energy Phys. 07 (2014) 079.

[14] R. D. Ball et al., Parton distributions with LHC data, Nucl. Phys. B867, 244 (2013).

[15] K. Kondo, Dynamical likelihood method for reconstruction of events with missing momentum. 2: Mass spectra for $2->2$ processes, J. Phys. Soc. Jpn. 60, 836 (1991).

[16] K. Kondo, T. Chikamatsu, and S. H. Kim, Dynamical likelihood method for reconstruction of events with missing momentum. 3: Analysis of a CDF high $\mathrm{p}(\mathrm{T})$ e mu event as $\mathrm{t}$ anti-t production, J. Phys. Soc. Jpn. 62, 1177 (1993).

[17] R. H. Dalitz and G. R. Goldstein, The decay and polarization properties of the top quark, Phys. Rev. D 45, 1531 (1992).

[18] R. H. Dalitz and G. R. Goldstein, Analysis of top-antitop production and dilepton decay events and the top quark mass, Phys. Lett. B 287, 225 (1992).

[19] G. R. Goldstein, K. Sliwa, and R. H. Dalitz, On observing top quark production at the tevatron, Phys. Rev. D 47, 967 (1993).

[20] R. H. Dalitz and G. R. Goldstein, Where is top?, Int. J. Mod. Phys. A 09, 635 (1994).

[21] V. Khachatryan et al. (CMS Collaboration), Study of hadronic event-shape variables in multijet final states in 
pp collisions at $\sqrt{s}=7 \mathrm{TeV}$, J. High Energy Phys. 10 (2014) 087.

[22] A. Banfi, G. P. Salam, and G. Zanderighi, Phenomenology of event shapes at hadron colliders, J. High Energy Phys. 06 (2010) 038.
[23] G. Aad et al. (ATLAS Collaboration), Measurement of three-jet production cross-sections in $p p$ collisions at $7 \mathrm{TeV}$ centre-of-mass energy using the ATLAS detector, Eur. Phys. J. C 75, 228 (2015). 